\title{
The Effect of Discharge Chamber Geometry on the Characteristics of Low-Pressure RF Capacitive Discharges
}

\author{
Valeriy A. Lisovskiy, Jean-Paul Booth, Karine Landry, David Douai, \\ Valerick Cassagne, and Vladimir D. Yegorenkov
}

\begin{abstract}
We report the measured extinction curves and current-voltage characteristics (CVCs) in several gases of RF capacitive discharges excited at $13.56 \mathrm{MHz}$ in chambers of three different geometries: 1) parallel plates surrounded by a dielectric cylinder ("symmetric parallel plate"); 2) parallel plates surrounded by a metallic cylinder ("asymmetric confined"); and 3) parallel plates inside a much larger metallic chamber ("asymmetric unconfined"), similar to the gaseous electronics conference reference cell. The extinction curves and the CVCs show differences between the symmetric, asymmetric confined, and asymmetric unconfined chamber configurations. In particular, the discharges exist over a much broader range of RF voltages and gas pressures for the asymmetric unconfined chamber. For symmetric and asymmetric confined discharges, the extinction curves are close to each other in the regions near the minima and at lower pressure, but at higher pressure, the extinction curve of the asymmetric confined discharge runs at a lower voltage than the one for the discharge in a symmetric chamber. In the particular cases of an "asymmetric unconfined chamber" discharge or "asymmetric confined" one, the RF discharge experiences the transition from a "weak-current" mode to a "strong-current" one at lower RF voltages than is the case for a "symmetric parallel-plate" discharge.
\end{abstract}

Index Terms-Chamber configuration, current-voltage characteristics (CVCs), extinction curve, RF capacitive discharge.

\section{INTRODUCTION}

$\mathbf{L}$ OW-PRESSURE RF capacitive discharges are widely used for etching and surface modification of various materials, for depositing oxides, nitrides and other films, for cleaning

Manuscript received November 18, 2005; revised November 23, 2006. This work was supported by the Unaxis France-Displays Division, Palaiseau, France.

V. A. Lisovskiy was with the Laboratoire de Physique et Technologie des Plasmas, Ecole Polytechnique, 91128 Palaiseau, France. He is now with the Department of Physics and Technology, Kharkov National University, 61077 Kharkov, Ukraine.

J.-P. Booth is with the Laboratoire de Physique et Technologie des Plasmas, Ecole Polytechnique, 91128 Palaiseau, France, and also with the Lam Research Corporation, Fremont, CA 94538 USA.

K. Landry is with the Unaxis, 38100 Grenoble, France.

D. Douai is with the Association Euratom-CEA, Département de Recherches sur la Fusion Contrôlée, CEA Cadarache, 13108 Saint Paul lez Durance, France.

V. Cassagne is with the Riber, 95873 Bezons, France.

V. D. Yegorenkov is with the Department of Physics, Kharkov National University, 61077 Kharkov, Ukraine.

Digital Object Identifier 10.1109/TPS.2007.893261 deposition chambers, in plasma chemistry, and for medical tool sterilization.

A great variety of $R \& D$ problems under solution and a large number of research groups occupied with studying the properties of RF capacitive discharge in experiment resulted in a series of discharge vessels differing not only in the electrode diameter and interelectrode gap but also in the design (geometry) of the vessels. Four types of discharge vessels are mostly applied. The parallel-plate RF discharge, in which flat electrodes are surrounded by a dielectric cylinder (in the case of a cylindrical shape with flat electrodes), is the simplest design [1]-[5]. Here, the discharge can burn only inside the interelectrode gap. The asymmetric confined RF discharge, where in the plane-parallel design of the electrodes a metal cylinder connected to one of the electrodes (conventionally to a grounded one) is used instead of a dielectric one, may be attributed to the second type [6]-[12]. The plasma enhanced chemical vapor deposition devices (aimed for depositing semiconductor and dielectric films), in which the vessel has a rectangular design, are attributed to the same type [13]-[16]. We can attribute to the third type the design when the flat electrodes (of comparatively small size) are introduced inside the grounded vessel of large dimensions [17]-[24]. The fourth type-gaseous electronics conference (GEC) reference cell-is similar to the third type (flat electrodes in a large vessel) [25]-[30]. In the vessels of the third and fourth types, the RF discharge can usually burn not only inside the gap between the flat electrodes but also outside it in a large vessel with grounded walls. Obviously, with the fixed values of the gas pressure, the RF field frequency, RF voltage amplitude across the electrodes, and the interelectrode gap RF discharge characteristics (discharge current, delivered power, inner plasma characteristics as well as the extinction curve of the discharge) in different vessels will not be identical.

In our recent paper [31], we presented the breakdown curves of the RF discharge in chambers of different geometry. Breakdown curves, of course, are very important for estimating the RF voltage values at which a gas breakdown occurs and a selfsustained discharge ignites. However, they give no information on the range of RF voltage and gas pressure values within which a discharge can be sustained. It is just an extinction curve that shows under which conditions a discharge burns in a chamber of certain geometry and when the technological processes can be performed. RF discharge extinction in a symmetric chamber was studied in rather sufficient detail (see [32] and papers cited 
therein). However, the available references do not contain the data on extinction curves of the RF discharge registered for identical electrodes placed in discharge chambers of different geometries.

The effect of chamber geometry on current-voltage characteristics (CVCs) and charge burning regimes remains not to be studied in full. In the papers [1]-[30] listed above, the research was performed with a fixed chamber geometry. Boeuf and Merad [33] obtained numerically the distributions of potential and ionization rate for GEC cell-like chamber and for asymmetric confined discharge. Beneking [2] registered the CVCs for two electrodeless discharge configurations in which the field-supplying electrodes were separated from the discharge volume by a dielectric wall, as well as for a symmetric parallel-plate chamber with inner electrodes.

The objective of this paper, therefore, is to study how the discharge chamber geometry affects the shape of the extinction curves, CVCs, and burning modes for RF capacitive discharges. We have studied three different chamber geometries: 1) parallel plates surrounded by a dielectric cylinder ("symmetric chamber"), 2) parallel plates surrounded by a metallic cylinder ("asymmetric confined chamber"), and 3) parallel plates inside a much larger metallic chamber ("asymmetric unconfined chamber"), similar to the GEC reference cell.

We observed that the symmetric, asymmetric confined, and unconfined chambers have different extinction curves and RF CVCs. For the asymmetric unconfined chamber, the discharge exists over a much broader range of RF voltages and gas pressures. In the case of the asymmetric confined discharge, the extinction curve at higher pressure runs below one for the discharge in the symmetric chamber. However, near the minima for the symmetric and asymmetric confined discharges and to the left of them, the extinction curves are close to each other. For the discharge transition to a strong current mode in the "symmetric parallel-plate" case, there are required higher RF voltages than those for "asymmetric unconfined chamber" and "asymmetric confined" cases.

\section{EXPERIMENTAL}

\section{A. Experimental Setups}

Experiments were performed for three different configurations, which we shall denominate as the following: 1) the symmetric chamber, 2) the asymmetric confined chamber, and 3 ) the asymmetric unconfined chamber.

The vacuum vessel consists of a $315-\mathrm{mm}$ diameter and 231-mm high steel chamber, with a view port to observe breakdown. Two parallel 143-mm diameter aluminum electrodes are installed in this vessel. The upper (powered) electrode is $10-\mathrm{mm}$ thick and is separated from a grounded shield (20-mm thick) by a layer of dielectric material. The lower (grounded) electrode is $30 \mathrm{~mm}$ thick. The gas was input through small holes in the upper (powered) electrode and evacuated via the external chamber. The gas pressure was monitored with capacitance manometers (10 and 1000 torr, MKS Instruments). A constant gas flow of $5 \mathrm{sccm}$ was set with a mass flow controller. The gas pressure was set by a feedback-controlled valve on the pumping outlet.

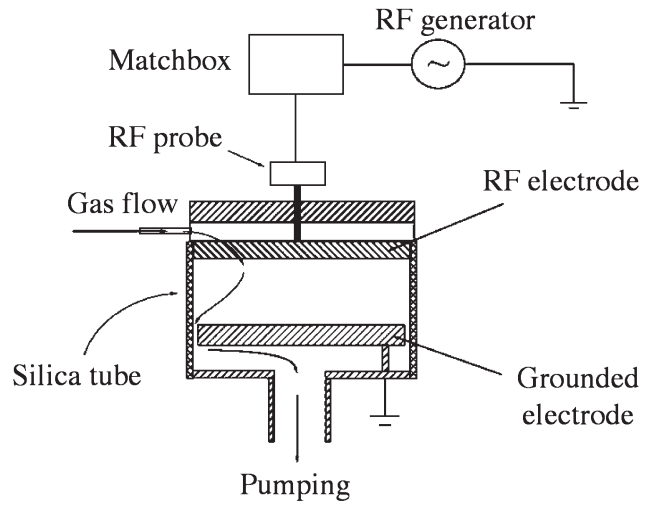

Fig. 1. Schematic of the symmetric RF discharge.

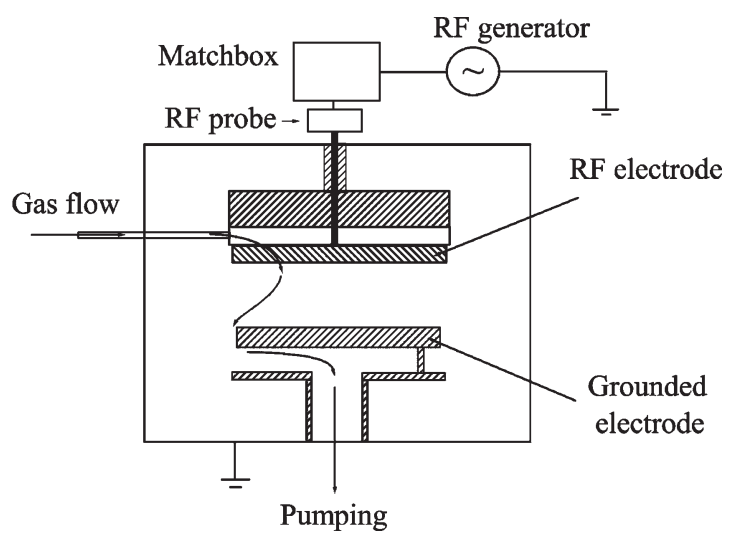

Fig. 2. Schematic of the asymmetric unconfined chamber configuration.

In the "symmetric chamber" configuration, the two electrodes were surrounded by a $145-\mathrm{mm}$ internal diameter fused silica tube, so that the effective discharge volume resembles Fig. 1. In the "asymmetric unconfined chamber" configuration (Fig. 2), the same electrodes were used, but the fused silica tube was removed so that the electrodes were located within a metallic chamber with grounded walls. Similar chamber configurations are widely used for studying the characteristics of the RF discharges (see, e.g., [17]-[24]). Experiments were performed in argon, nitrogen, and hydrogen over the pressure range $p \approx 0.01-10$ torr with an RF field frequency of $f=13.56 \mathrm{MHz}$. The extinction curves and the CVCs of the discharge were recorded for interelectrode gap values of $L=11.9 \mathrm{~mm}$ and $L=27 \mathrm{~mm}$.

"Symmetric chamber" was also used to achieve the third "asymmetric confined chamber" configuration (Fig. 3). In this case, the inner surface of the discharge tube was covered with a grounded aluminum foil. A gap of $2 \mathrm{~mm}$ was left between the foil edge and the surface of the RF electrode. Similar chambers (from the viewpoint of the asymmetric distribution of the vacuum RF field) have also been widely used in other studies of RF discharges [6]-[16].

\section{B. Electrical Measurements}

An RF voltage-current probe (Advanced Energy Z'SCAN) was used to measure the RF voltage $U_{\mathrm{rf}}$, the RF current $I_{\mathrm{rf}}$, 


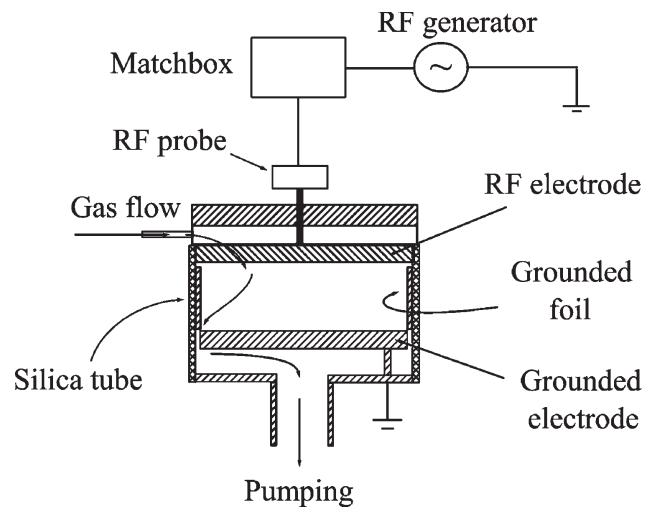

Fig. 3. Schematic of the asymmetric confined chamber, with foil on the radial walls. (a)

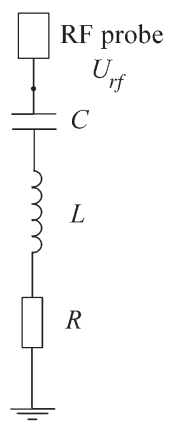

(b)

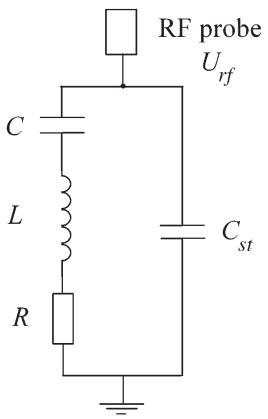

Fig. 4. Equivalent circuit (a) for the symmetric RF discharge and (b) for the discharge with a large stray capacitor (asymmetric unconfined chamber).

the phase shift $\varphi$ between the RF voltage and RF current and the delivered power. It was situated as close as possible to the $\mathrm{RF}$ electrode. The RF power (13.56 MHz) was supplied by an RF generator (RF Power Products Inc. RF5S) via an L-type matchbox (Huttinger Elektronik Gmbh PFM).

In an asymmetric unconfined chamber (Fig. 3), the RF voltage-current probe was located outside a large grounded chamber, and the electrodes were inside it. In this case, a large stray capacitance between the RF electrode and the grounded chamber walls affects the measurements of electric characteristics of the discharge considerably made with an external RF voltage-current probe (RF current amplitude $I_{\mathrm{rf}}$, phase shift angle $\varphi$ between the RF voltage and current, and discharge impedance $Z$ ). In order to decrease the effect of the stray capacitance on electric measurements, Miller and co-workers [34], [35] and Sobolewski [25], [36] proposed to include an external shunt into the discharge circuit, thus removing a large displacement current and allowing to obtain more accurate values of the current $I_{\mathrm{rf}}$, phase shift angle $\varphi$, and discharge impedance $Z$.

Let us consider the way the stray capacitance affects the electric measurements. Fig. 4(a) shows the equivalent circuit of the RF discharge in a symmetric chamber, where $R$ and $L$ are the resistance and inductance of the discharge plasma and $C$ is the capacitance of the near-electrode sheaths. For the given circuit, the impedance $Z_{0}$, the current amplitude $I_{0}$, the phase shift angle $\varphi_{0}$, and the active current $I_{0} \cdot \cos \varphi_{0}$ for the symmetric chamber are equal to

$$
\begin{aligned}
\left|Z_{0}\right| & =\sqrt{R^{2}+\left(\omega L-\frac{1}{\omega C}\right)^{2}} \\
I_{0} & =\frac{U_{\mathrm{rf}}}{\left|Z_{0}\right|}=U_{\mathrm{rf}} / \sqrt{R^{2}+\left(\omega L-\frac{1}{\omega C}\right)^{2}} \\
\cos \varphi_{0} & =R / \sqrt{R^{2}+\left(\omega L-\frac{1}{\omega C}\right)^{2}} \\
I_{0} \cdot \cos \varphi_{0} & =U_{\mathrm{rf}} \cdot R /\left[R^{2}+\left(\omega L-\frac{1}{\omega C}\right)^{2}\right]
\end{aligned}
$$

where $U_{\mathrm{rf}}$ is the RF voltage amplitude and $\omega=2 \pi f$ is the angular frequency of the RF electric field.

For the equivalent circuit of the discharge with the asymmetric unconfined chamber [Fig. 4(b)] possessing a large stray capacitance $C_{\mathrm{st}}$, we have the following expressions:

$$
\begin{aligned}
|Z| & =\left[R^{2}+\left(\omega L-\frac{1}{\omega C}\right)^{2}\right] / Q \\
\cos \varphi & =R / Q \\
I & =U_{\mathrm{rf}} \cdot Q /\left[R^{2}+\left(\omega L-\frac{1}{\omega C}\right)^{2}\right] \\
Q & =\sqrt{R^{2}+\left\{\omega C_{\mathrm{st}}\left[R^{2}+\left(\omega L-\frac{1}{\omega C}\right)^{2}\right]-\left(\omega L-\frac{1}{\omega C}\right)\right\}^{2}} \\
I \cdot \cos \varphi & =U_{\mathrm{rf}} \cdot R /\left[R^{2}+\left(\omega L-\frac{1}{\omega C}\right)^{2}\right] \\
& =I_{0} \cdot \cos \varphi_{0} .
\end{aligned}
$$

It follows from formulas (1)-(9) that the stray capacitance $C_{\mathrm{st}}$ affects the measurements of the impedance $Z$, RF current amplitude $I_{\mathrm{rf}}$, and phase shift angle $\varphi$, but does not affect the measurements of the active current $I_{\mathrm{rf}} \cdot \cos \varphi$, as well as of the delivered power

$$
P_{\mathrm{dlv}}=\frac{1}{2} U_{\mathrm{rf}} I_{\mathrm{rf}} \cos \varphi
$$

The same conclusion may be drawn considering the results by Sobolewski [25, Table II]. Without the external shunt, he obtained $I_{\mathrm{rf}}=1.816 \mathrm{~A}$ and $\varphi=-88.3^{\circ} \pm 0.6^{\circ}$, whereas in its presence, he got $I_{\mathrm{rf}}=0.24 \mathrm{~A}$ and $\varphi=-75.7^{\circ} \pm 0.6^{\circ}$. Then, without the shunt, we have $I_{\mathrm{rf}} \cdot \cos \varphi=53.9 \mathrm{~mA}$ (more precisely, taking into account the error in measuring the phase shift, the active current values were within the range of 35$66.6 \mathrm{~mA}$ ). With the shunt, we have $I_{\mathrm{rf}} \cdot \cos \varphi=59.3 \mathrm{~mA}$ (more precisely, within the range of 56.8-61.7 mA). Thus, the value of the active RF current with the shunt and without it happened to be constant to the accuracy of measuring the phase shift angle. The values of the delivered power [25] also almost coincided: without the shunt, $P_{\mathrm{dlv}}=2.66 \mathrm{~W}$, whereas 


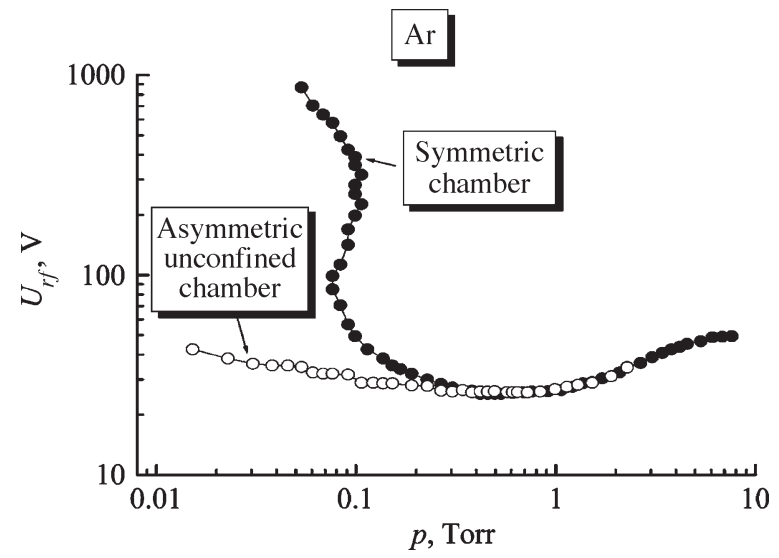

Fig. 5. Extinction curves of the RF discharge in argon at $L=11.9 \mathrm{~mm}$ : Full circles depict the results for the symmetric discharge; empty circles depict the results for the asymmetric unconfined chamber, respectively.

with it, $P_{\mathrm{dlv}}=2.88 \mathrm{~W}$. In our paper studying the regimes of discharge burning in chambers of different geometry, we were primarily interested in registering the active RF current, but not the current amplitude, phase shift angle, or impedance separately; therefore, we did not apply an external shunt in our measurements.

\section{RESULTS}

Let us consider how the discharge chamber design affects the extinction curves and the CVCs of an RF discharge. The region of existence of a discharge is limited by its extinction curve. The extinction curves of an RF discharge in argon are shown in Fig. 5. For pressures greater than 0.4 torr, the extinction curves for the symmetric and asymmetric unconfined chambers coincide and possess a single minimum. However, at a lower pressure, the extinction curve for the symmetric chamber deviates abruptly to higher voltages and even possesses a multivalued section. Similar behavior of the extinction curves of the symmetric discharge was also observed in [32]. The RF voltage for extinction in the asymmetric unconfined chamber increases, but only slowly, as the argon pressure is lowered. For a tenfold decrease of the argon pressure (from 0.2 to 0.02 torr), the extinction RF voltage increases by only $10 \mathrm{~V}$.

For nitrogen (Fig. 6), the extinction curves of the symmetric and asymmetric unconfined chambers coincide for pressures above 1 torr. As the pressure is lowered, the extinction curve of the symmetric chamber, after passing through the minimum, deviates to higher RF voltages. At the same time, the extinction curve in the asymmetric unconfined chamber, after passing the minimum, drops to lower RF voltages and approaches a second minimum at $p \approx 0.05$ torr.

Figs. 5 and 6 show that, at low gas pressure, a broad range of RF voltages exists within which a discharge cannot be sustained in the symmetric chamber, but may exist in the asymmetric unconfined chamber. For example, in Fig. 6, we see that a discharge can be sustained in the asymmetric unconfined chamber with a nitrogen pressure of 0.05 torr and an RF voltage of $60 \mathrm{~V}$, whereas a discharge cannot exist in the symmetric chamber at this RF voltage for any pressure.

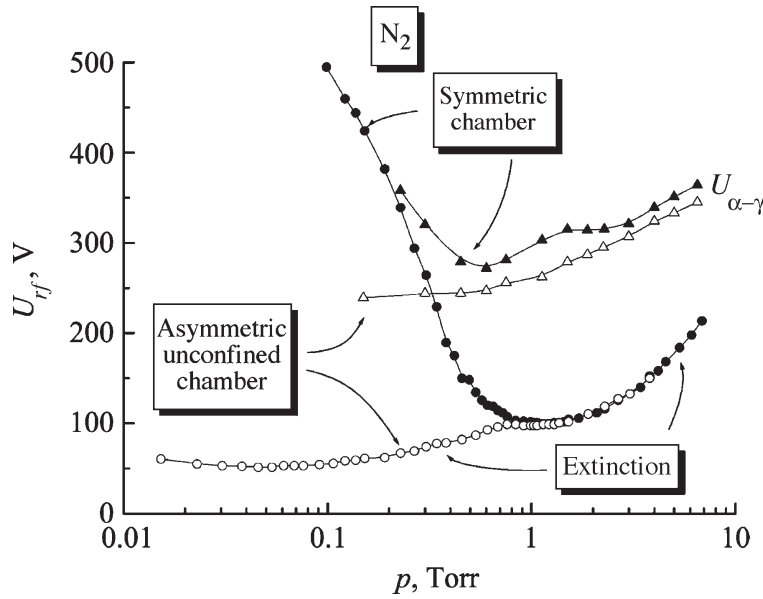

Fig. 6. Extinction curves of the RF discharge in nitrogen at $L=11.9 \mathrm{~mm}$ : Full circles depict the results for the symmetric discharge; empty circles depict the results for the asymmetric unconfined chamber, respectively. The $\alpha-\gamma$ transition curves for the RF discharge $\left(U_{\alpha \gamma}\right)$ for the symmetric (full triangles) and asymmetric unconfined chambers (empty triangles).

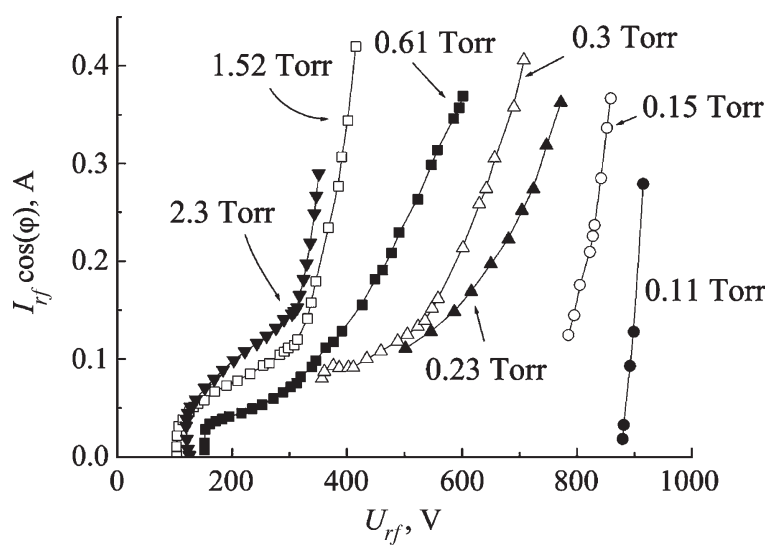

Fig. 7. Active RF current against RF voltage for the symmetric RF discharge at different nitrogen pressures, $L=11.9 \mathrm{~mm}$.

Now, let us consider the CVCs of discharges in the two chambers. Specifically, we shall consider the dependence of the active RF current $I_{\mathrm{rf}} \cdot \cos \varphi$ on the RF voltage. The experiments were performed for nitrogen at $L=11.9 \mathrm{~mm}$. Fig. 7 shows the CVC for the symmetric chamber. For gas pressures less than 0.15 torr, a small increase in the RF voltage causes a rapid increase of the active RF current, and the discharge is always in a high-current mode. At higher gas pressure, the rate of increase of the RF current is somewhat lower, and a weak-current mode exists at low voltage (in agreement with the conclusions in [4] and [37], the weak-current mode cannot exist at too low gas pressure). Dogleg features are clearly observed, indicating the transition from the weak-current $(\alpha-)$ mode to the strong-current $(\gamma-)$ one. Usually, the discharge covers the entire surface of the electrodes, except at pressures above 0.8 torr and low RF voltages when the discharge was in the weak-current "normal" discharge regime.

Fig. 8 shows the CVC of the asymmetric unconfined discharge (with the electrodes in the chamber). At low pressure ( $p \approx 0.01$ torr), the discharge occurred solely in the large chamber (outside of the electrode gap) over the whole range 


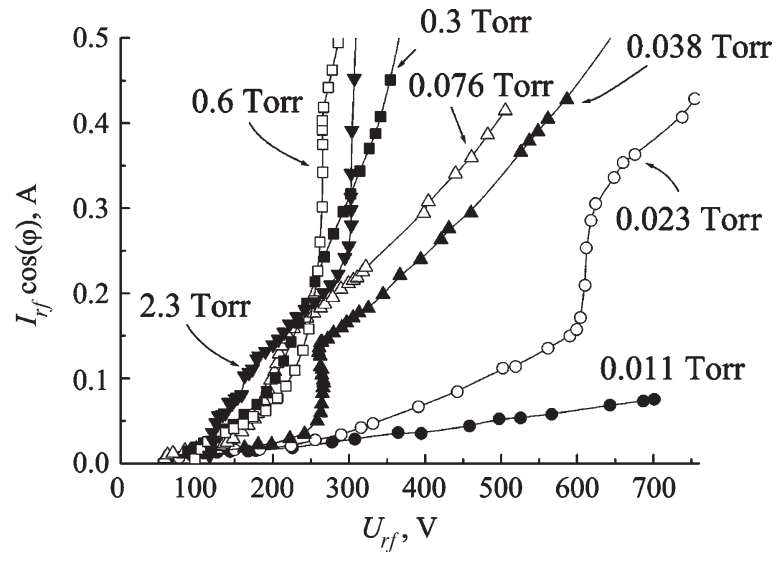

Fig. 8. Active RF current against RF voltage for the asymmetric unconfined chamber at different nitrogen pressures, $L=11.9 \mathrm{~mm}$.

of the RF voltage studied. At higher gas pressure, as the RF voltage is increased, the discharge occurred first in the large chamber and, then, (at a sufficiently high RF voltage) also penetrated the gap between the planar electrodes. This was accompanied by an abrupt increase of the active RF current. Such a situation was observed, for example, at $p \approx 0.023$ torr and $U_{\text {rf }} \approx 600 \mathrm{~V}$, and at $p \approx 0.038$ torr and $U_{\text {rf }} \approx 260 \mathrm{~V}$ (see Fig. 8). At a pressure of $\sim 0.1$ torr, the discharge was ignited both in the outer chamber and in the interelectrode gap. For pressures below 1 torr, the discharge in the gap is nonuniform across the electrode surface, having a toroidal shape located close to the radial boundaries of the electrodes. On increasing the RF voltage and gas pressure, the discharge glow expanded toward the electrode axis. In the strong-current mode, a bright glow was observed only near the boundary of the RF electrode sheath, whereas the glow was weak at the grounded electrode (as well as near the planar surface of the electrode and other grounded parts of the large chamber). However, at higher pressure ( $p>1$ torr), the glow intensity near the grounded surfaces increases. At $p>1$ torr, the gas breakdown occurs in the gap between the electrodes. The discharge at the smallest voltages (near the extinction voltage) was in the normal regime having the same toroidal shape. With an increasing RF voltage, the discharge also expanded into the outer chamber and transformed into the anomalous mode. Nonuniform radial distribution of plasma parameters in GEC cell was predicted in theory in [33] and [38], whereas the paper of Overzet and Hopkins [26] demonstrated in experiment the presence of the plasma density maximum near the radial boundary of electrodes in agreement with the results of our paper.

Fig. 9 compares the CVCs for the symmetric and asymmetric unconfined chambers at $p=0.3$ torr and $p=1.52$ torr. The smallest RF voltage that could maintain a discharge at $p=$ 0.3 torr was about $350 \mathrm{~V}$, whereas the discharge in the asymmetric unconfined chamber was already in the strong-current mode. At a nitrogen pressure $p=1.52$ torr, the discharges in both the symmetric and asymmetric unconfined chambers had approximately the same extinction voltage, and at the smallest voltages, they operated in the normal regime, with similar values of the discharge current. However, the active current of the discharge in the asymmetric unconfined chamber increased

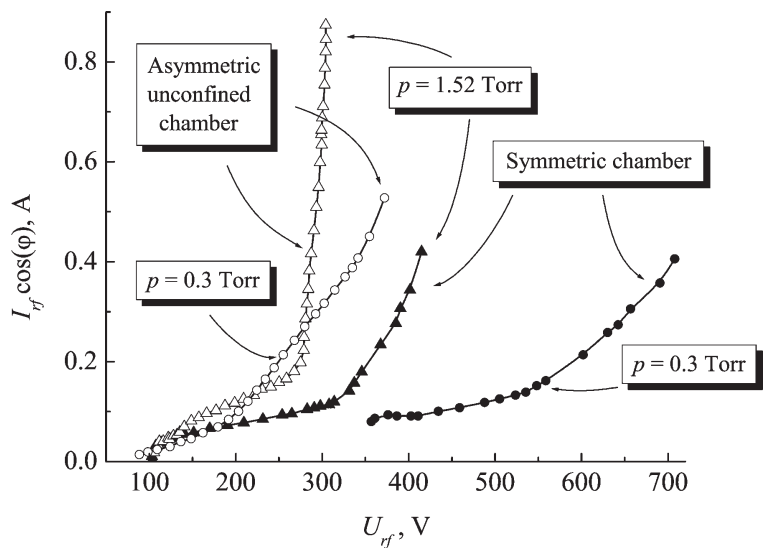

Fig. 9. Active RF current against RF voltage at nitrogen pressure $p=0.3$ torr (full and empty circles) and $p=1.52$ torr (full and empty triangles) for the symmetric RF discharge (full circles and triangles) and for the asymmetric unconfined chamber (empty circles and triangles), gap width $L=11.9 \mathrm{~mm}$.

faster than that in the symmetric chamber. At $U_{\alpha \gamma} \approx 279 \mathrm{~V}$, the transition to the strong-current mode occurred, and then, the discharge current increased rapidly. The active current of the discharge in the symmetric chamber increased much more slowly with the RF voltage in both the weak-current and strongcurrent modes, and an RF voltage $U_{\alpha \gamma} \approx 314 \mathrm{~V}$ was required to accomplish the $\alpha-\gamma$ transition.

In Fig. 6, apart from the extinction curves, we also depict the $\alpha-\gamma$ transition curves of the RF discharge $\left(U_{\alpha \gamma}\right)$ for the symmetric and asymmetric unconfined chambers. In a symmetric chamber with gas pressure decreasing the magnitude, $U_{\alpha \gamma}$ first decreases and, at $p \approx 2$ torr, approaches a minimum. At this section under $\alpha-\gamma$ transition, there occurs a breakdown of the near-electrode sheath via electrons produced from the electrode surface through a bombardment with positive ions and acquired a sufficiently large energy while moving in the RF field in the sheath. The quantity $U_{\alpha \gamma}$ at the minimum $(314 \mathrm{~V})$ is close to the minimum voltage for a dc breakdown curve $(284 \mathrm{~V}$, [39]); the value of the product $p d$ also is in agreement with it (in case of the dc breakdown, $d$ is the gap between plane electrodes $p d \approx 0.65$ torr $\cdot \mathrm{cm}[39]$; in the case of the $\alpha-\gamma$ transition, $d=d_{\text {sh }}$ is the thickness of the nearelectrode sheath, $p d \approx 0.7$ torr $\cdot \mathrm{cm}$ ).

With further pressure decrease, the RF voltage of the $\alpha-\gamma$ transition decreases approaching the second minimum, and then, the $\alpha-\gamma$ transition curve coincides with the extinction curve of the RF discharge (such behavior of the $\alpha-\gamma$ transition curve was observed earlier in papers [4], [37]). In this section, the $\alpha-\gamma$ transition occurs without a breakdown of the nearelectrode sheath; the breakdown criterion is not met in this case [4], [37]. But at the same time, the sheath becomes a source of fast electrons capable of ionizing the gas molecules in their path. The lower is the gas pressure, the higher is the probability of the process that secondary electrons leaving the surface of the electrodes under bombardment with ions, metastable atoms, and photons, will traverse the near-electrode sheath experiencing one or several collisions with gas molecules, or even actually without collisions. The gas pressure affects the velocity of secondary electrons accelerated in the near-electrode sheath. At low gas pressure, an electron may acquire more energy, 
seldom experiencing collisions with the gas molecules. At high pressure, the electrons often collide with the gas molecules and acquire less energy after crossing the near-electrode sheath. The electrons can acquire over the total sheath thickness the energy up to $\varepsilon_{e} \approx e U_{\mathrm{sh}}$ (where $U_{\mathrm{sh}}$ is the RF voltage drop across the sheath), and a beam of fast electrons travels from the sheath into the plasma. Under a lower gas pressure, the RF discharge can exist only in $\gamma$-mode, because even with the lowest RF voltage sustaining the discharge, the voltage drop across the near-electrode sheath is sufficiently large for the energy of fast electrons leaving the sheath to attain the ionization potential of the gas molecules.

The $\alpha-\gamma$ transition curve for the asymmetric unconfined chamber, with a gas pressure decreasing uniformly, decreases, approaching at a lower pressure a minimum value of $\approx 240 \mathrm{~V}$. At the same time, for the asymmetric unconfined chamber, the RF voltage of the $\alpha-\gamma$ transition was always less than the one for the symmetric chamber.

Obviously, the self-bias voltage affects the discharge characteristics. In the asymmetric chamber, the area of the RF electrode is much less than the one of the grounded surfaces; the RF electrode acquires the dc negative potential $U_{\mathrm{dc}}$ with respect to the grounded one [40], [41]. This dc potential increases the dc voltage drop across the near-electrode sheath, accelerating the positive ions moving out of the plasma to the RF electrode. The ions possessing a higher energy bombard the electrode surface and hit a larger number of secondary electrons [42]. In an asymmetric chamber, the potential of the RF electrode equals $-U_{\mathrm{dc}}+U_{\mathrm{rf}} \cdot \sin \omega t$. Then, neglecting the RF voltage drop across the quasi-neutral plasma, we get the maximum instant voltage drop across the sheath near the RF electrode $\left|U_{\mathrm{dc}}+U_{\mathrm{rf}}\right|$. Consequently, in an asymmetric chamber (confined and unconfined), the presence of the dc self-bias voltage at the RF electrode facilitates the breakdown of the near-electrode sheath and the discharge transition from the weak-current mode to the strong-current one.

There are also other reasons leading to the decrease of the $\mathrm{RF}$ voltage of the $\alpha-\gamma$ transition in the asymmetric unconfined chamber. Usually, the discharge ignites not only between the planar electrodes but also in the whole chamber (except in the normal regime at sufficiently high gas pressure). At low gas pressure $(p<0.5$ torr $)$, in the weak-current discharge mode, the plasma in the large chamber shines brightly, indicating the high density of plasma outside the gap. Electrons and ions flow from the large chamber into the gap between the planar electrodes and compensate for the loss of charged particles to the electrodes (in the symmetric chamber, electrons and ions are lost to the electrodes and dielectric walls of the discharge chamber). The flux of photons from the discharge in the large chamber may also excite and ionize the gas molecules within the interelectrode gap, as well as induce an enhanced emission of electrons from the surface of the electrodes. Long-lived metastable atoms and molecules entering the gap may lead to a multistep ionization as well as to the appearance of additional secondary electrons from the surface of the electrodes. Therefore, the discharge in the asymmetric unconfined chamber experiences a transition from $\alpha$ - to $\gamma$-mode at remarkably less $\mathrm{RF}$ voltage values than in the symmetric chamber.

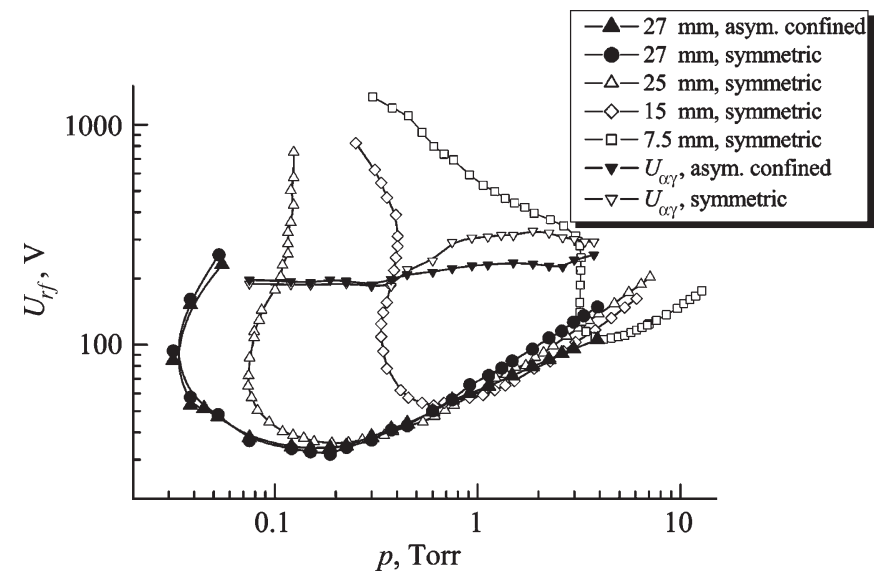

Fig. 10. Extinction curves of the RF discharge in hydrogen: Full circles depict the results for the asymmetric confined chamber $(L=27 \mathrm{~mm})$; full triangles $(L=27 \mathrm{~mm})$, empty triangles $(L=25 \mathrm{~mm})$, empty diamonds $(L=$ $15 \mathrm{~mm})$, and empty squares $(L=7.5 \mathrm{~mm})$ depict the results for the symmetric discharge. The $\alpha-\gamma$ transition curves of the RF discharge $\left(U_{\alpha \gamma}\right)$ for the symmetric (solid upside-down triangles) and asymmetric confined chambers (empty upside-down triangles), $L=27 \mathrm{~mm}$.

Let us now discuss how the asymmetry of the discharge affects its conditions of existence and the CVCs. Fig. 10 shows the extinction curves of the RF discharge in hydrogen at $L=27 \mathrm{~mm}$ for the "symmetric" and "asymmetric confined" discharges. It is clear from the figure that the extinction curves of the RF discharge actually coincide near the minima and to the left of them. However, at a higher pressure ( $p>0.7$ torr), the extinction voltage for the asymmetric confined case is lower than the one for the symmetric case, and the spacing between the curves increases with pressure.

The weak-current RF discharge in an asymmetric confined chamber at sufficiently high pressure $(p>1$ torr) is burning before extinction in the form of a small plasma bunch between the RF electrode and the surface of the grounded foil, whereas in the central region of the gap, the discharge is extinct. Sharp boundaries of the sheaths are observed only in the small region of contact between the plasma and the surfaces of the RF electrode and foil. Perhaps, it is just the region where the ionization via electron impact occurs, which is necessary for discharge sustainment, i.e., the self-sustained discharge is burning. The charged particles formed within the bunch enter the rest of the gap surrounding this region due to diffusion, the boundaries of the sheaths being not sharp (or smeared) here. Here, the gas ionization rate is small; the plasma is in a state of decay, and further from the bunch, the discharge glow disappears. The burning discharge has the shape of an arc standing on the mutually perpendicular surfaces of the RF electrode and the grounded foil. (We indeed observed the discharge glow behavior described in the preceding item as follows. Having finished the registration of the extinction curves and CVCs of the discharge in an asymmetrically confined chamber, we made a vertical slit in the grounded foil around $1-\mathrm{cm}$ wide. It enabled us to observe the process of discharge extinction in this chamber. This additional slit had no effect on the magnitude of the extinction voltage. It was difficult to perform the accurate optical measurements of, say, the plasma bunch diameter before extinction, but a general behavior of the discharge glow was clearly seen.) The 
width (cross section) of this arc is comparatively not large, and the near-electrode regions at an enlarged pressure are thin and quasi-planar. Therefore, we can attempt to explain the behavior of the extinction curve in the asymmetric confined chamber by using the extinction curves in the symmetric chamber. Fig. 10 depicts the extinction curves in the symmetric chamber as well as in the asymmetric confined chamber (both were registered for $L=27 \mathrm{~mm}$ ), as well as the extinction curves in the symmetric chamber for $L=25,15$, and $7.5 \mathrm{~mm}$. As shown in Fig. 10, the extinction curve in the asymmetric confined chamber is actually an envelope for the family of extinction curves in the symmetric chamber, registered for lesser distances between the electrodes, than is the gap in the asymmetric confined chamber.

It is clear in Fig. 10 that the more interelectrode spacing there is, the lower is the extinction curve minimum of the $\mathrm{RF}$ discharge. Therefore, for the interelectrode gap of $25 \mathrm{~mm}$, the minimum voltage for the discharge sustainment is approximately $35 \mathrm{~V}$. Decreasing the gap leads not only to the increase of the minimum voltage for discharge sustainment but also to the shift of the extinction curve to the region of higher gas pressure. The extinction curve for the symmetric discharge for the gap of $15 \mathrm{~mm}$ starts to run below the extinction curve for the gap of $25 \mathrm{~mm}$ just at the hydrogen pressure $p \geq 0.7$ torr (see Fig. 10). Consequently, at low pressure, the symmetric and asymmetric confined discharges before extinction are burning between the flat electrodes; the extinction curves for them are identical. However, with an increasing gas pressure, it becomes more profitable for the asymmetric confined discharge to burn not only between the flat electrodes but also between the flat surface of the RF electrode and the surface of the grounded foil (discharge chamber wall). For this lesser gap, the voltage for the discharge sustainment is lower than that for the gap between the flat surfaces of the electrodes. Therefore, the RF discharge in the asymmetric confined chamber before extinction at high pressure tends to be located closer to the grounded surface of the discharge tube (and the more is the gas pressure, the closer the discharge glow is located to the RF electrode), and the extinction curve for the asymmetric confined discharge runs below one for the symmetric discharge.

On decreasing the gap $L$, the extinction curves for the symmetric chamber are shifted to higher RF voltage and gas pressure values in such a way that they cross each other. Extinction curves in the symmetric chamber were studied in detail in a recent paper [32]. Let us explain this behavior of the extinction curves as follows. Let us fix the gas pressure and vary the gap between the electrodes registering the voltage of the discharge extinction. When the gap is very narrow, the total width of the near-electrode sheaths is only somewhat less than the interelectrode gap. In this case, the losses of charge particles to the electrodes are high, and the RF voltage drop across the narrow region of the quasi-neutral plasma is small. Therefore, a sufficiently high RF voltage is required for discharge sustainment. For a larger interelectrode gap, the escape of the charged particles to the electrodes decreases; the discharge is extinguished at a lesser RF voltage. However, with the further increase of the interelectrode gap, the RF voltage drop across the regions of quasi-neutral plasma increases; therefore, the RF voltage at which the discharge is extinct approaches a minimum

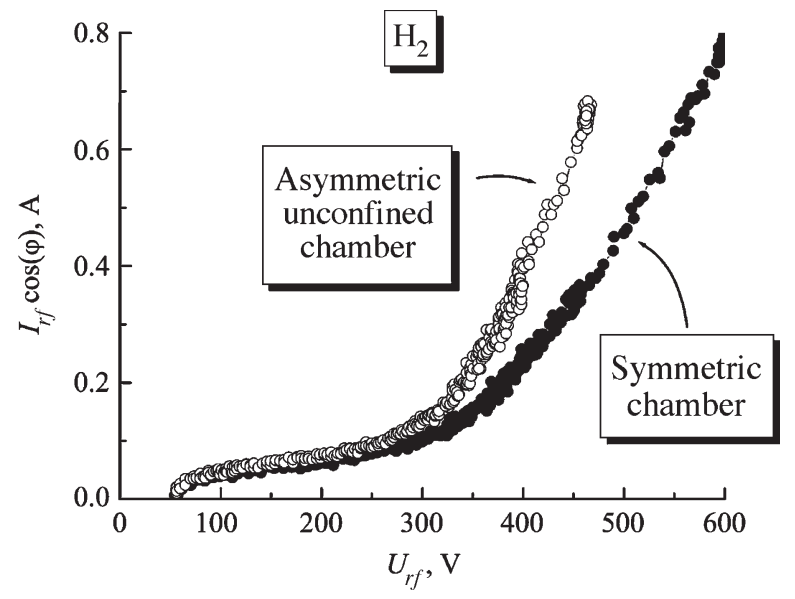

Fig. 11. Current-voltage characteristics of the RF discharge in hydrogen at $L=27 \mathrm{~mm}$ and $p=0.75$ torr: Full circles depict the results for the symmetric discharge; empty circles depict the results for the asymmetric confined discharge, respectively.

and then grows. The location of this minimum is determined by the gas pressure. For a higher gas pressure, the thickness of the near-electrode sheaths is smaller, and the thickness of the plasma region is bigger; therefore, the minimum will be observed at a smaller distance between the electrodes. As for a fixed gas pressure, the minimum value of the RF discharge extinction voltage exists at some gap value $L_{1}$, then for a larger gap $L_{2}>L_{1}$, the RF extinction voltage value will be larger than one for $L_{1}$. Therefore, the extinction curves $U_{\mathrm{rf}}(p)$ for two different gap values $L_{1}$ and $L_{2}$ mutually intersect at a certain point, the extinction curve for a smaller gap being shifted to the region of higher RF voltage and gas pressure values.

Fig. 11 shows the CVCs of the RF discharge in hydrogen at $L=27 \mathrm{~mm}$ and $p=0.75$ torr. It is clear from the figure that in the weak-current $(\alpha)$ mode, the CVC of the asymmetric confined discharge runs a little above the CVC of the symmetric discharge. However, in the strong-current $(\gamma)$ mode, the discharge current through the discharge chamber happens to be much higher than the one through the symmetric chamber. At the same time, the $\alpha-\gamma$ transition voltage in the asymmetric confined chamber was less than the one in the symmetric one (256 and $292 \mathrm{~V}$ for the asymmetric confined and symmetric chambers, respectively).

Fig. 10 also shows the curves of the $\alpha-\gamma$ transition $\left(U_{\alpha \gamma}\right)$ of the RF discharge for the symmetric and asymmetric confined chambers. It follows from the figure that at low gas pressure, these curves actually coincide, and the existence region of the weak-current mode of the RF discharge is limited from the lowpressure side by the extinction curve. At low gas pressure, the quasi-neutral plasma in the symmetric and asymmetric confined chambers shines uniformly, with the best conditions for the discharge sustainment, probably, existing near the chamber axis between the flat electrodes. On increasing gas pressure, the curve of the $\alpha-\gamma$ transition for the asymmetric confined chamber runs at considerably lower voltage values than for the symmetric chamber. It is probably due to the presence of the region of higher plasma density between the surface of the grounded foil and the RF electrode: Just here, the discharge in the asymmetric confined chamber shines as the brightest of 
all. The presence of such region with an enhanced ionization rate was predicted numerically earlier in paper [33]. Near this region, the near-electrode sheath is remarkably thinner, and an enhanced flow of positive ions is falling on the surfaces of the RF electrode and the foil [33], thus making easier the discharge transition to the strong-current mode.

\section{CONCLUSION}

Technological processes, e.g., for the anisotropic etching of semiconductors at low gas pressure, are often performed in RF discharges with the electrodes inside a large chamber over a broad range of gas pressure. However, it should be kept in mind that the symmetric RF discharges and the discharges with the electrodes inside a large chamber (such as the GEC reference cell) possess different characteristics.

The following conclusions may be drawn from all this. Discharges in the chambers comprising the electrodes inside a large chamber differ considerably from the symmetric RF discharges. The RF discharge between the planar parallel electrodes limited with a dielectric tube and the RF discharge with identical planar electrodes placed inside a discharge chamber of larger diameter with grounded metallic walls possess different extinction curves and CVCs. In the latter case, the discharge may exist over a much broader range of gas pressure and RF voltage values. The extinction curves and the CVCs exhibit differences for the symmetric as well as asymmetric discharge chambers, especially in the range of high gas pressure.

\section{ACKNOWLEDGMENT}

The authors would like to thank the Unaxis FranceDisplays Division, Palaiseau, France, for the equipment used in this paper.

\section{REFERENCES}

[1] L. J. Overzet, J. H. Beberman, and J. T. Verdeyen, "Enhancement of the negative ion flux to surfaces from radio-frequency processing discharges," J. Appl. Phys., vol. 66, no. 4, pp. 1622-1631, Aug. 1989.

[2] C. Beneking, "Power dissipation in capacitively coupled RF discharges," J. Appl. Phys., vol. 68, no. 9, pp. 4461-4473, Nov. 1990.

[3] V. A. Godyak, R. B. Piejak, and B. M. Alexandrovich, "Electrical characteristics of parallel-plate RF discharges in argon," IEEE Trans. Plasma Sci., vol. 19, no. 4, pp. 660-676, Aug. 1991.

[4] V. A. Lisovskiy, "Features of the $\alpha-\gamma$ transition in a low-pressure rf argon discharge," Tech. Phys., vol. 43, no. 5, pp. 526-534, May 1998.

[5] H. Ikegaki, S. Tajima, M. Endo, and H. Amemiya, "Efficiency of parallelplate capacitive radio frequency discharge plasma," Jpn. J. Appl. Phys., vol. 41, no. 7A, pp. 4729-4730, Jul. 2002.

[6] J. D. P. Passchier and W. J. Goedheer, "A two-dimensional fluid model for an argon rf discharge," J. Appl. Phys., vol. 74, no. 6, pp. 3744-3751, Sep. 1993.

[7] O. Leroy, P. Stratil, J. Perrin, J. Jolly, and P. Belenguer, "Spatiotemporal analysis of the double layer formation in hydrogen radio frequency discharges," J. Phys. D, Appl. Phys., vol. 28, no. 3, pp. 500-507, Mar. 1995.

[8] G. J. Nienhuis, W. J. Goedheer, E. A. G. Hamers, W. G. J. H. M. van Sark, and J. Bezemer, "A self-consistent fluid model for radio-frequency discharges in $\mathrm{SiH}_{4}-\mathrm{H}_{2}$ compared to experiments," J. Appl. Phys., vol. 82, no. 5, pp. 2060-2071, Sep. 1997.

[9] O. Leroy, G. Gousset, L. L. Alves, J. Perrin, and J. Jolly, "Twodimensional modelling of $\mathrm{SiH}_{4}-\mathrm{H}_{2}$ radio-frequency discharges for a-Si:H deposition," Plasma Sources Sci. Technol., vol. 7, no. 3, pp. 348-358, Aug. 1998.
[10] M. Hertl and J. Jolly, "Laser-induced fluorescence detection and kinetics of $\mathrm{SiH}_{2}$ radicals in $\mathrm{Ar} / \mathrm{H}_{2} / \mathrm{SiH}_{4} \mathrm{RF}$ discharges," J. Phys. D, Appl. Phys., vol. 33, no. 4, pp. 381-388, Feb. 2000.

[11] N. Chaabane, A. V. Kharchenko, H. Vach, and P. Roca i Cabarrocas, "Optimization of plasma parameters for the production of silicon nanocrystals," New J. Phys., vol. 5, no. 1, pp. 37.1-37.15, Apr. 2003.

[12] A. V. Kharchenko, V. Suendo, and P. Roca i Cabarrocas, "Plasma studies under polymorphous silicon deposition conditions," Thin Solid Films, vol. 427, no. 1, pp. 236-240, Mar. 2003.

[13] J. Schmitt, M. Elyaakoubi, and L. Sansonnens, "Glow discharge processing in the liquid crystal display industry," Plasma Sources Sci. Technol., vol. 11, no. 3A, pp. A206-A210, Aug. 2002.

[14] M. A. Lieberman, J. P. Booth, P. Chabert, J. M. Rax, and M. M. Turner, "Standing wave and skin effects in large-area, high-frequency capacitive discharges," Plasma Sources Sci. Technol., vol. 11, no. 3, pp. 283-293, Aug. 2002.

[15] L. Sansonnens, J. Bondkowski, S. Mousel, J. P. M. Schmitt, and V. Cassagne, "Development of a numerical simulation tool to study uniformity of large area PECVD film processing," Thin Solid Films, vol. 427, no. 1, pp. 21-26, Mar. 2003.

[16] H. Schmidt, L. Sansonnens, A. A. Howling, C. Hollenstein, M. Elyaakoubi, and J. P. M. Schmitt, "Improving plasma uniformity using lens-shaped electrodes in a large area very high frequency reactor," J. Appl. Phys., vol. 95, no. 9, pp. 4559-4564, May 2004.

[17] N. Mutsukura, K. Kobayashi, and Y. Machi, "Monitoring of radiofrequency glow-discharge plasma," J. Appl. Phys., vol. 66, no. 10, pp. 4688-4695, Nov. 1989

[18] F. Tochikubo, T. Kokubo, S. Kakuta, A. Suzuki, and T. Makabe, "Investigation of the high-frequency glow discharge in $\mathrm{Ar}$ at $13.56 \mathrm{MHz}$ by spatiotemporal optical emission spectroscopy," J. Phys. D, Appl. Phys., vol. 23, no. 9, pp. 1184-1192, Sep. 1990.

[19] G. Oelerich-Hill, I. Pukropski, and M. Kujawka, "On the characterization of a RF parallel plate discharge," J. Phys. D, Appl. Phys., vol. 24, no. 4, pp. 593-601, Apr. 1991.

[20] B. M. Annaratone, V. P. T. Ku, and J. E. Allen, "Identification of plasmasheath resonances in a parallel-plate plasma reactor," J. Appl. Phys., vol. 77, no. 10, pp. 5455-5457, May 1995.

[21] C. A. Anderson and W. G. Graham, "Temporally and spatially resolved plasma parameters and EEDF measurements in a low-pressure discharge," Plasma Sources Sci. Technol., vol. 4, no. 4, pp. 561-570, Nov. 1995.

[22] N. Spiliopoulos, D. Mataras, and D. E. Rapakoulias, "Power dissipation and impedance measurements in radio-frequency discharges," J. Vac. Sci. Technol. A, Vac. Surf. Films, vol. 14, no. 5, pp. 2757-2765, Sep. 1996.

[23] T. Kitajima, M. Izawa, N. Nakano, and T. Makabe, "The time-resolved two-dimensional profile of a radiofrequency capacitively coupled plasma," J. Phys. D, Appl. Phys., vol. 30, no. 12, pp. 1783-1789, Jun. 1997.

[24] S. J. You, H. C. Kim, C. W. Chung, H. Y. Chang, and J. K. Lee, "Mode transition for power dissipation induced by driving frequency in capacitively coupled plasma," J. Appl. Phys., vol. 94, no. 12, pp. 7422-7426, Dec. 2003.

[25] M. A. Sobolewski, "Electrical characterization of radio-frequency discharges in the gaseous electronics conference reference cell," J. Vac. Sci. Technol. A, Vac. Surf. Films, vol. 10, no. 6, pp. 3550-3562, Nov. 1992.

[26] L. J. Overzet and M. B. Hopkins, "Spatial variations in the charge density of argon discharges in the gaseous electronics conference reference reactor," Appl. Phys. Lett., vol. 63, no. 18, pp. 2484-2486, Nov. 1993.

[27] S. Djurovic, J. R. Roberts, M. A. Sobolewski, and J. K. Olthoff, "Absolute spatially and temporally-resolved optical emission measurements of $\mathrm{rf}$ glow discharges in argon," J. Res. Natl. Inst. Stand. Technol., vol. 98, no. 2, pp. 159-180, 1993.

[28] J. K. Olthoff and K. E. Greenberg, "The gaseous electronics conference reference cell-An introduction," J. Res. Natl. Inst. Stand. Technol., vol. 100 , no. 4, pp. 327-339, 1995.

[29] L. Lauro-Taroni, M. M. Turner, and N. S. J. Braithwaite, "Analysis of the excited argon atoms in the GEC RF reference cell by means of onedimensional PIC simulations," J. Phys. D, Appl. Phys., vol. 37, no. 16, pp. 2216-2222, Aug. 2004.

[30] M. A. Sobolewski, "Experimental test of models of radio-frequency plasma sheaths," Appl. Phys. Lett., vol. 70, no. 8, pp. 1049-1051, Feb. 1997.

[31] V. Lisovskiy, S. Martins, K. Landry, D. Douai, J.-P. Booth, V. Cassagne, and V. Yegorenkov, "The effect of discharge chamber geometry on the ignition of low-pressure RF capacitive discharges," Phys. Plasmas, vol. 12, no. 9, pp. 093 505.1-093 505.8, Sep. 2005. 
[32] V. Lisovskiy, J.-P. Booth, S. Martins, K. Landry, D. Douai, and V. Cassagne, "Extinction of RF capacitive low-pressure discharges," Europhys. Lett., vol. 71, no. 3, pp. 407-411, Aug. 2005.

[33] J. P. Boeuf and A. Merad, "Fluid and hybrid models of non equilibrium discharges," in Plasma Processing of Semiconductors, vol. 336, P. F. Williams, Ed. Boston, MA: Kluwer, 1997, pp. 291-320.

[34] P. A. Miller, "Electrical characterization of RF plasmas," Proc. SPIE, vol. 1594, pp. 179-188, 1991.

[35] P. A. Miller, H. Anderson, and M. P. Splichal, "Electrical isolation of radio-frequency plasma discharges," J. Appl. Phys., vol. 71, no. 3, pp. 1171-1176, Feb. 1992

[36] M. A. Sobolewski, "Electrical characteristics of argon radio frequency glow discharges in an asymmetric cell," IEEE Trans. Plasma Sci., vol. 23, no. 6, pp. 1006-1022, Dec. 1995.

[37] V. A. Lisovskiy and V. D. Yegorenkov, "Alpha-gamma transition in RF capacitive discharge in low-pressure oxygen," Vacuum, vol. 74, no. 1, pp. 19-28, 2004.

[38] D. P. Lymberopoulos and D. J. Economou, "Modeling and simulation of glow discharge plasma reactors," J. Vac. Sci. Technol. A, Vac. Surf. Films, vol. 12, no. 4, pp. 1229-1236, Jul. 1994.

[39] V. A. Lisovskiy, S. D. Yakovin, and V. D. Yegorenkov, "Low-pressure gas breakdown in uniform dc electric field," J. Phys. D, Appl. Phys., vol. 33, no. 21, pp. 2722-2730, Nov. 2000.

[40] J. W. Coburn and E. Kay, "Positive-ion bombardment of substrates in rf diode glow discharge sputtering," J. Appl. Phys., vol. 43, no. 12, pp. 4965-4971, Dec. 1972.

[41] K. Kohler, J. W. Coburn, D. E. Horne, and E. Kay, "Plasma potentials of 13.56-MHz rf argon glow discharges in a planar system," J. Appl. Phys., vol. 57, no. 1, pp. 59-66, Jan. 1985.

[42] A. V. Phelps and Z. L. Petrovic, "Cold-cathode discharges and breakdown in argon: Surface and gas phase production of secondary electrons," Plasma Sources Sci. Technol., vol. 8, no. 3, pp. R21-R44, Aug. 1999.

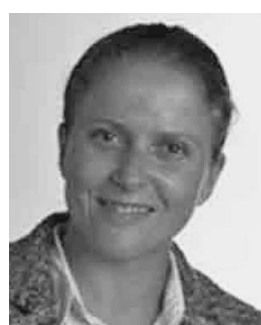

Karine Landry received the B.S. and M.S. degrees in physics from Université Joseph Fourier, Grenoble, France, in 1989 and 1991, respectively, and the Ph.D. degree in materials science and engineering from the Institut National Polytechnique de Grenoble, Grenoble, in 1995.

From 1995 to 1998, she was with the University of Wisconsin, Madison, as a Postdoctoral Research Associate. From 1999 to 2005, she was with the Unaxis Displays Division France SAS, Palaiseau, France, successively as an, R\&D Engineer, Project Leader, and Team Leader. She is currently with Unaxis, Grenoble as an R\&D Project Manager

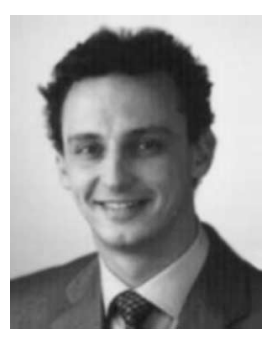

David Douai was born in Antony, France, in 1969. He received the degree in physics from Paris XI University, Orsay, France and the Ph.D. degree in plasma physics from Ruhr Universität Bochum, Germany, in 2001.

In 2001, he assisted public and industrial end users to develop specific PAPVD projects with Lille University of Sciences and Technology, France. In 2002, he joined the Research and Development Center of Unaxis Displays, Palaiseau, France, as a Senior Research Engineer on RF PECVD (plasma enhanced chemical vapor deposition) systems for the flat panel displays industry. $\mathrm{He}$ is currently working as a research scientist on Tokamaks wall conditioning systems at the French Atomic Energy Commission (CEA).

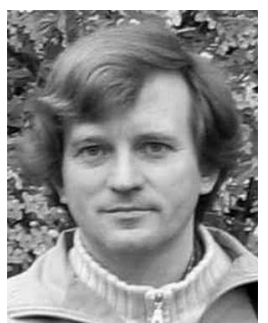

Valeriy A. Lisovskiy was born in Donetsk region, Ukraine, in August 1964. He received the M.Sc. degree in physics and the Ph.D. degree in physics and chemistry of plasmas from Kharkov State University, Kharkov, Ukraine, in 1990 and 1993, respectively.

From 2001 to 2004, he was with the Ecole Polytechnique, Palaiseau, France, as a Postdoctoral Research Associate. He is currently an Associate Professor with Kharkov National University, Kharkov. His research interests include low-pressure RF, LF, dc, combined and dual-frequency gas discharges, plasma processing, electron transport in gases, and plasma sterilization.

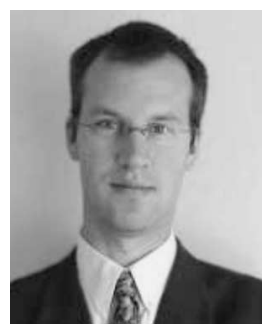

Valerick Cassagne was born in France, in 1968. $\mathrm{He}$ received the M.S. and Ph.D. degrees in mechanics and material science from Ecole Nationale Supérieure d'Arts et Métiers, Paris.

He has worked for nine years with Balzers in the development of PVD and PECVD equipment and processes for flat panel displays industry, leading the $\mathrm{R} \& \mathrm{D}$ center in the last position. He is currently a Business Development Director with Riber, Bezons, France, MBE equipment manufacturer.

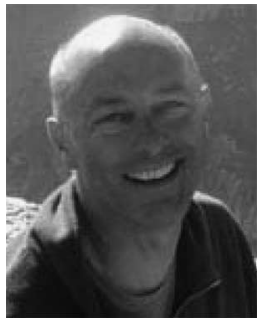

Jean-Paul Booth received the D.Phil. degree in physical chemistry from Oxford University, Oxford, U.K., in 1988.

After receiving the D.Phil. degree, he then worked with the France-Telecom CNET Research Centre, Meylan, France, before entering the Centre National de la Recherche Scientifique, Grenoble, France. In 2000, he moved to the Ecole Polytechnique, Palaiseau, France, to found the Radio-Frequency Plasmas in Molecular Gases Group, Laboratoire de Physique et Technologie des Plasmas. He is also with the Lam Research Corporation, Fremont, CA. His research interests are centered on optical (including laser) and electrical diagnostics of reactive plasmas. He has a particular interest in radio frequency excited plasmas in fluorocarbon gases used for $\mathrm{SiO}_{2}$ etching in VLSI manufacture. He is the author of more than 50 refereed journal articles and three review articles and is the holder of two patents. He has instigated and organized six international workshops on fluorocarbon plasma science.

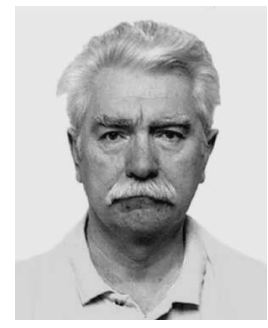

Vladimir D. Yegorenkov was born in 1944. He received the M.Sc. degree in physics, the Ph.D. and Dr.Sc. degrees in physics and chemistry of plasmas from Kharkov State University, Kharkov, Ukraine, in 1968, 1974, and 1992, respectively.

$\mathrm{He}$ is currently a Full Professor with Kharkov National University, Kharkov. His research interests concentrate on the studies of physics of fusion plasmas and gas discharge, general physics, and lecture demonstration experiments in physics. 